\title{
A single recessive mutation in the proteolytic machinery of Arabidopsis chloroplasts impairs photoprotection and photosynthesis upon cold stress
}

Received: 26 May 2003 / Accepted: 6 September 2003 / Published online: 25 October 2003

(C) Springer-Verlag 2003

\begin{abstract}
Intracellular proteases, together with molecular chaperones, are components of the cellular protein quality control system. Although the identity of chloroplast proteases has been revealed in recent years, little is known about their regulation. As a first step towards identifying unknown functional or regulatory components of the chloroplast proteolytic machinery, a genetic screen was devised with the aim of generating Arabidopsis thaliana (L.) Heynh. mutants impaired in chloroplast protein degradation. A streptomycin-resistance gene was fused to a cDNA construct that encodes an unstable mutant of the OE33 protein. This chimeric gene was used for transforming Arabidopsis plants. Analysis of transgenic plants revealed a correlation between streptomycin resistance and accumulation of the recombinant fusion protein. Seeds from transgenic plants that were sensitive to streptomycin were chemically mutagenized and screened for resistance to streptomycin. Such resistance could be due to stabilization of the protein caused by a mutation in the chloroplast proteolytic machinery. Genetic analysis of one of the mutants showed the mutation to be recessive, in a single nuclear gene. Further characterization of the mutant revealed that it was not a result of increased transcription of the
\end{abstract}

M. Levy $(\bowtie) \cdot$ Z. Adam

The Robert H. Smith Institute of Plant Sciences

and Genetics in Agriculture,

The Faculty of Agriculture,

The Hebrew University of Jerusalem,

76100 Rehovot, Israel

E-mail: levy@vegmail.ucdavis.edu

Fax: + 1-530-7529659

A. Bachmair

Institute of Botany, University of Vienna, 1030 Vienna, Austria

Present address: M. Levy

Department of Vegetable Crops,

University of California, Davis, CA 95616, USA

Present address: A. Bachmair

Department of Developmental Biology of Plants,

Max Planck Institute for Breeding Research,

50829 Cologne, Germany transgene. Moreover, chloroplast lysates from the mutant plant showed decreased ATP-dependent degradation of a chloroplast protein substrate, consistent with the conclusion that the mutation impaired the proteolytic machinery. Physiological analysis revealed that upon cold stress, photoprotection and photosynthesis in the mutant was inhibited, suggesting that the chloroplast proteolytic machinery is involved in repairing damage incurred to the photosynthetic machinery upon exposure to cold temperatures.

Keywords Arabidopsis . Chloroplast .

Photoprotection · Photosynthesis · Proteolysis

Abbreviations $N P Q$ : non-photochemical quenching - OE33: the 33-kDa subunit of the oxygenevolving complex $\cdot R I S P$ : Rieske Fe-S protein $\cdot T^{R}$ : antibiotic-resistant transgenic plants $\cdot T^{S}$ : antibioticsensitive transgenic plants

\section{Introduction}

Cellular proteases play an indispensable role in all living organisms. Their two major functions are to regulate the level of key regulatory proteins (Gottesman 1996; Hershko and Ciechanover 1998) and, together with molecular chaperones, to provide a protein quality control system for the cell (Gottesman et al. 1997; Wickner et al. 1999). These two functions have been demonstrated in both eukaryotic and prokaryotic cells, and components of the prokaryotic proteolytic machinery have been found and characterized in eukaryotic organelles such as mitochondria (Langer and Neupert 1996; Langer 2000) and chloroplasts (Adam 1996, 2000).

Proteolytic degradation processes in chloroplasts were documented long before the identities of the proteases involved were discovered. Increased degradation rates were observed especially in response to exposure to high light intensities, under conditions that led to photoinhibition of photosynthesis (for review, see Adam 
1996; Andersson and Aro 1997). At least in the case of the D1 protein of photosystem II, proteolytic degradation is an essential step in a repair mechanism that allows replacement of a photodamaged and therefore inactive subunit with a newly synthesized one (Prasil et al. 1992; Andersson and Aro 1997; Melis 1999). Numerous examples of degradation of proteins lacking their prosthetic groups or missing their complex partners have also been documented (for review, see Adam 1996).

The identity of chloroplast proteases has started to become available in the last decade, primarily as a result of molecular and biochemical approaches. Three main families of chloroplast proteases are now known (for review, see Adam and Clarke 2002). Clp protease is an ATP-dependent serine protease which is a hetero-multimer complex composed of the proteolytic subunit ClpP and the regulatory ATPase $\mathrm{ClpC}$, and is located in the stroma (Shanklin et al. 1995; Clarke 1999), where it functions in the degradation of unassembled soluble and membrane proteins (Halperin and Adam 1996; Majeran et al. 2000; Halperin et al. 2001). FtsH protease is a membrane-bound ATP-dependent metalloprotease, the ATP-binding and catalytic domains of which are exposed to the stromal side of the thylakoid membrane (Lindahl et al. 1996). It has been implicated in the degradation of unassembled proteins in thylakoid membranes and stroma (Ostersetzer and Adam 1997), and in the degradation of photodamaged D1 protein of photosystem II (Lindahl et al. 2000). FtsH has also been suggested to be involved in the biogenesis of thylakoid membranes, as mutants affected at an $\mathrm{fts} H$ gene were linked to a variegated phenotype (Chen et al. 2000; Takechi et al. 2000). The third family of chloroplast proteases is DegP. It is an ATP-independent serine protease that has been found peripherally associated with thylakoids. Two such proteases have been identified to date. One faces the lumenal side of the thylakoid membrane (Itzhaki et al. 1998; Chassin et al. 2002), whereas the other is oppositely oriented, facing the stroma (Haussuhl et al. 2001). This second isomer was recently identified as the protease that mediates the initial cleavage of photodamaged D1 protein into two fragments (Haussuhl et al. 2001), enabling their further degradation by FtsH (Lindahl et al. 2000).

As sequencing of the Arabidopsis thaliana genome progressed, it became evident that the aforementioned proteases occur in multiple isomers in plant cells. Six genes encoding ClpP, 2 encoding ClpC, 11 encoding $\mathrm{FtsH}$, and 13 encoding DegP have been identified (Adam et al. 2001; Peltier et al. 2001). The products of most of these genes are expected to be targeted to chloroplasts. Despite these advances in elucidating components of the proteolytic machinery in chloroplasts, regulation of this machinery is largely unknown. Thus, we designed a genetic screen aimed at generating Arabidopsis mutants impaired in the degradation of chloroplast proteins. Such mutants can, in principle, be affected at either a protease gene or any regulatory function that affects proteolysis. Here we describe the generation of these mutant plants, and the molecular, biochemical, genetic and physiological characterization of one of them.

\section{Materials and methods}

\section{Constructs, vectors and bacteria}

To create the "Translation Construct", a mutant OE33 construct whose product is mistargeted to the chloroplast stroma (Halperin and Adam 1996), was used. Its $3^{\prime}$ end (without the stop codon) was fused to the $5^{\prime}$ end of the bacterial aadA gene (Tait et al. 1985), which confers resistance to streptomycin, to create a translational fusion (see Fig. 1). A 961-bp HincII-ClaI fragment containing the aadA gene was ligated in-frame into the respective sites in the mutant OE33 construct. This fusion construct was then subcloned into the SacI-ClaI sites of the pSP72 vector, under control of the SP6 promoter. In-vitro-coupled transcription/translation of this construct was carried out using a commercially available kit (Promega), to yield a radiolabeled precursor protein. The "Transformation Construct" was created in two steps. First, the enhancer region of the CaMV $35 \mathrm{~S}$ promoter was duplicated in the plasmid pRT103 (Topfer et al. 1987) by insertion of a HincII-EcoRV fragment of pRT103 into the HincII site of pRT103. The double

\section{Translation Construct}

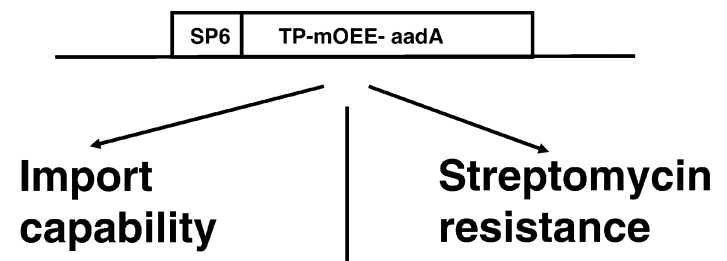

Transformation Construct

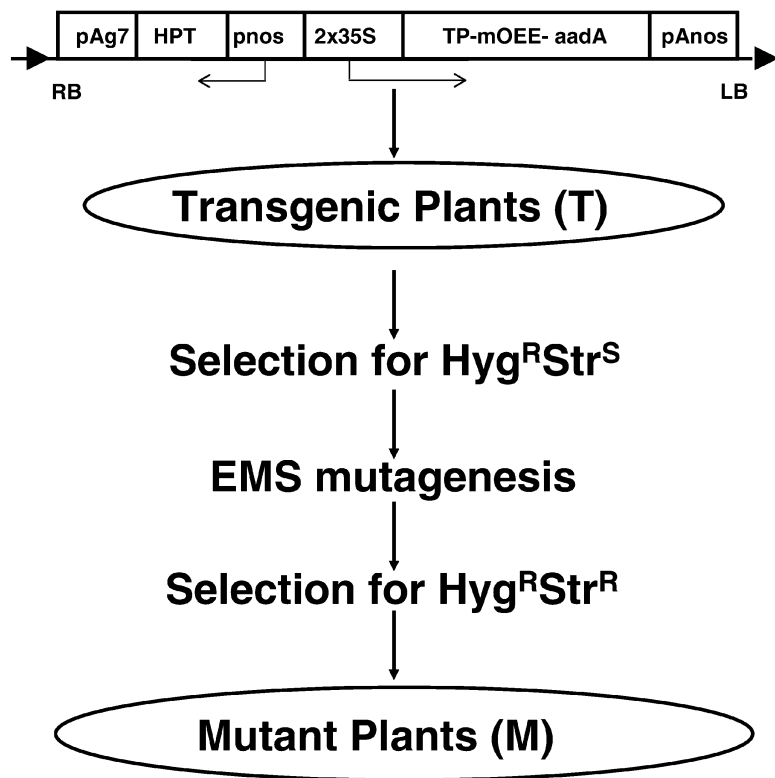

Fig. 1 Strategy for the generation of Arabidopsis thaliana mutants impaired in degradation of chloroplast proteins. A schematic presentation of the strategy is outlined. Hyg hygromycin; Str, streptomycin; ${ }_{R}$, resistant; $S$, sensitive; $E M S$, ethyl-methane sulfonate; $p A g 7$, terminator; $p A n o s$, terminator; pnos, promotor (for other construct components, see text) 
CaMV promoter from this plasmid was inserted into the HindIII site of the pBIB-Hyg vector (Becker 1990) in an outward-reading orientation. Then, the $X h o \mathrm{I}-\mathrm{XbaI}$ fragment from the "Translation Construct" containing the coding region of the fusion protein, was subcloned behind the double CaMV promoter (see Fig. 1). Plasmid amplification was carried out in Escherichia coli DH5 $\alpha$ cells. For generation of bacterial cells for plant transformation, Agrobacterium tumefaciens strain GV3101, containing the pMP90RK plasmid (Koncz et al. 1989), was transformed with the binary plasmid containing the "Transformation Construct". These cells were then used for plant transformation (see below).

Plant material, transformation and mutagenesis

Plants of Arabidopsis thaliana (L.) Heynh., ecotype Col-o, were grown at $22{ }^{\circ} \mathrm{C}$, at a light intensity of $100 \mu \mathrm{mol}$ photons $\mathrm{m}^{-2} \mathrm{~s}^{-1}$. For vegetative growth, plants were kept under an $8 \mathrm{~h}$ light $/ 16 \mathrm{~h}$ dark photoperiod. For reproductive growth, plants were grown under $14 \mathrm{~h}$ light $/ 10 \mathrm{~h}$ dark. Plants were transformed using the vacuum-infiltration method, as previously described (Bechtold et al. 1993), using 0.5× MS salts containing 3\% sucrose as a transformation medium. Transgenic seedlings were selected on agar plates containing $1 \times$ MS salts, $1 \times$ Gamborg vitamin mix and $5 \mathrm{mg}$ $\mathrm{ml}^{-1}$ pure agar (all from Sigma), and $20 \mu \mathrm{g} \mathrm{ml} \mathrm{m}^{-1}$ hygromycin. Chemical random mutagenesis was performed on 30,000 seeds of a selected transgenic plant (designated 6T), by soaking them in $0.2 \%$ ethyl-methane sulfonate (EMS) for $8 \mathrm{~h} . \mathrm{M}_{1}$ seeds were germinated on soil and $\mathrm{M}_{2}$ seeds were harvested. These seeds were selected for growth on plates containing streptomycin $\left(50 \mu \mathrm{g} \mathrm{ml}^{-1}\right)$ and hygromycin $\left(20 \mu \mathrm{g} \mathrm{ml}^{-1}\right)$. Resistant plants were self-fertilized and their seeds were subjected to another round of antibiotic-resistance testing. For genetic analysis, mutants were subjected to reciprocal crosses with wild-type plants. $F_{2}$ progeny were tested on plates containing both streptomycin and hygromycin to assess dominance, and to determine whether the mutation was nuclear.

Polymerase chain reaction (PCR), reverse transcription

(RT)-PCR, RNA and protein blot analyses

DNA was isolated from plants as previously described (Krysan et al. 1996). To detect the transgene in transgenic plants, PCR was carried out with the following primers: either aadAforward-5'GCATTGCTCAATGACCTTATG3' or OE33-forward-5'GGGCATATGCCAAAGAGATTGACCTATGAT3', and aadA-reverse - 5'TCATTTACCAACTGACTTGAT3'. Reaction mixtures were subjected to $94{ }^{\circ} \mathrm{C}$ for $2 \mathrm{~min}$, followed by 35 cycles of $92{ }^{\circ} \mathrm{C}$ for $1.5 \mathrm{~min}, 55^{\circ} \mathrm{C}$ for $2 \mathrm{~min}$, and $72{ }^{\circ} \mathrm{C}$ for $2 \mathrm{~min}$. For RNA analysis, total RNA was isolated from plants using the RNeasy kit (Qiagen), and subjected to either RT-PCR or northern blot analyses. RT-PCR was carried out using the Promega kit, according to the manufacturers instructions, with the aadA-forward and aadA-reverse primers. For northern blot analysis, $3.3 \mu \mathrm{g}$ of total RNA was denatured in $50 \%$ formamide and $18 \%$ formaldehyde, separated on a $1.5 \%$ agarose-formaldehyde gel, and transferred to a Hybond-N + membrane (Amersham). The blot was probed with the cDNA for OE33 labeled with $\left[{ }^{32} \mathrm{P}\right] \mathrm{dCTP}$ using a random priming kit (Boehringer). Hybridization was carried out at $65^{\circ} \mathrm{C}$, the membrane was washed three times with 2,1 and $0.5 \times$ SSC, respectively, and then exposed to an X-ray film. For western blot analysis, protein samples were resolved by means of SDSPAGE on gels containing $12 \%$ acrylamide, blotted onto nitrocellulose membranes, and reacted with an antibody against OE33 (Itzhaki et al. 1998) at a dilution of 1:10,000. Blots were then crossreacted with goat anti-rabbit $\mathrm{IgG}$ conjugated to alkaline phosphatase (BioRad), and immune complexes were visualized using an enhanced chemiluminescence kit (Pierce). To allow good visualization of both the high-abundance OE33 and the low-abundance fusion protein, membranes were cut and the lower and upper parts, respectively, were developed separately.
Chloroplasts isolation and degradation assay

Chloroplasts were isolated from 2- to 3-week-old Arabidopsis plants. The leaves were ground in a Polytron blender in ice-cold grinding buffer [ $450 \mathrm{mM}$ sorbitol, $20 \mathrm{mM}$ Tricine $/ \mathrm{KOH}$ (pH 8.4), $5 \mathrm{mM}$ EGTA, $5 \mathrm{mM}$ EDTA, $10 \mathrm{mM} \mathrm{Na}_{2} \mathrm{CO}_{3}, 0.1 \%$ (w/v) BSA, $330 \mathrm{mg} \mathrm{l}^{-1}$ isoascorbate]. The suspension was filtered through two layers of Miracloth (Calbiochem), and centrifuged for $5 \mathrm{~min}$ at 5,000 rpm in a JA10 rotor (Beckman). The pellet was resuspended in grinding buffer and the suspension was layered onto a step gradient consisting of 70 , 50 and $40 \%(\mathrm{v} / \mathrm{v})$ Percoll [in a solution containing 1\% BSA, $1 \%$ Ficoll, 3\% (v/v) PEG, $450 \mathrm{mM}$ sorbitol, $1 \mathrm{mM} \mathrm{MgCl}_{2}, 1 \mathrm{mM}$ $\mathrm{MnCl}_{2}, 50 \mathrm{mM}$ Tricine ( $\mathrm{pH}$ 8.4)]. The gradient was centrifuged for $15 \mathrm{~min}$ at 5,000 rpm in an HB-4 rotor (Sorvall). The interface between the 50 and $70 \%$ layers was collected, diluted with 10 volumes of grinding buffer, and the chloroplasts were centrifuged for $3 \mathrm{~min}$ at $5,000 \mathrm{rpm}$ in a JA20 rotor (Beckman). Finally, the pellet was gently resuspended in grinding buffer.

To assess proteolytic activity, isolated chloroplasts were lysed in $10 \mathrm{mM}$ Hepes-KOH (pH 8.0) and incubated with $1,000 \mathrm{cpm}$ radiolabeled in-vitro-translated Rieske $\mathrm{Fe}-\mathrm{S}$ protein (RISP), in the presence or absence of $5 \mathrm{mM} \mathrm{Mg-ATP}$ for 2 PAGE, and gels were stained with $\mathrm{h}$ at $37^{\circ} \mathrm{C}$. The reaction mixtures were then subjected to SDS-PAGE, and gels were stained with Coomassie blue to verify equal loading, dried and exposed to a PhosphorImager screen (Fuji).

\section{Confocal microscopy}

Cotyledons from Arabidopsis plants were fixed for $2 \mathrm{~h}$ in $8 \%(\mathrm{v} / \mathrm{v})$ formaldehyde and $5 \%(\mathrm{v} / \mathrm{v})$ dimethyl sulfoxide (DMSO), in PEM buffer (100 mM PIPES, $5 \mathrm{mM}$ EDTA, $5 \mathrm{mM} \mathrm{MgCl}_{2}$ ). They were then washed three times in PEM buffer, treated with $2 \%$ driselase for $0.5 \mathrm{~h}$ and air-dried. Rehydration was performed in a PEM buffer with $0.03 \%(\mathrm{v} / \mathrm{v})$ Nonidet NP40, followed by three washes in PEM buffer. Samples were then examined with a confocal microscope (TCS 4D; Leica, Bensheim, Germany). The laser line of an argon-krypton laser was set to $568 \mathrm{~nm}$ combined with a beam splitter and a long-pass emission filter suitable for visualizing green excited autofluorescence of chloroplasts.

\section{Chlorophyll fluorescence measurement}

Chlorophyll fluorescence was measured at room temperature in attached rosette leaves, using a Hansatech MFMS/2S modulated fluorescence system (Hansatech Instruments). Minimal chlorophyll fluorescence $\left(F_{0}\right)$ was measured after $10 \mathrm{~min}$ of dark adaptation. The intensity of modulated measuring light was $0.3 \mu \mathrm{mol}$ photons $\mathrm{m}^{-2} \mathrm{~s}^{-1}$. Maximal chlorophyll fluorescence $\left(F_{\mathrm{M}}\right)$ was measured during a brief pulse of saturating non-modulated light from a halogen lamp (Hansatech PLS1; 3,000 $\mu \mathrm{mol}$ photons $\mathrm{m}^{-2} \mathrm{~s}^{-1}$ ). Steady-state chlorophyll fluorescence $\left(F_{\mathrm{S}}\right)$ was measured after $10 \mathrm{~min}$ of non-modulated actinic light adaptation (Hansatech LS2; natural density-filtered 440 or $770 \mu \mathrm{mol}$ photons $\left.\mathrm{m}^{-2} \mathrm{~s}^{-1}\right)$. Maximal chlorophyll fluorescence after light adaptation $\left(F_{\mathrm{MS}}\right)$ was measured during a brief pulse of saturating non-modulated light, exactly as after dark adaptation. The photosynthetic parameters that were calculated from the measured chlorophyll fluorescence were as follows: the maximum quantum yield of photosystem II electron transport: $F_{\mathrm{V}} / F_{\mathrm{M}}=\left(F_{\mathrm{M}}-F_{\mathrm{o}}\right) / F_{\mathrm{M}}$; quantum yield efficiency of photosystem II: $\mathrm{Y}=\left(F_{\mathrm{MS}}-F_{\mathrm{S}}\right) / F_{\mathrm{MS}}$; and non-photochemical quenching: $\mathrm{NPQ}=\left(F_{\mathrm{M}}-F_{\mathrm{MS}}\right) / F_{\mathrm{MS}}$.

\section{Results}

\section{The strategy}

To create Arabidopsis mutants that are impaired in their ability to degrade chloroplast proteins, we designed a 
two-step strategy based on positive selection. We previously demonstrated that when the lumenal protein OE33 is mistargeted to the stroma, it becomes unstable (Halperin and Adam 1996), a process that could be attributed to Clp protease (Halperin et al. 2001). Thus, we planned to fuse this construct to a streptomycinresistance gene (aadA), under the control of a CaMV $35 \mathrm{~S}$ promoter with duplicated enhancer region for constitutive expression. This construct would then be subcloned into a binary transformation vector, containing a hygromycin-resistance gene (HPT) under the control of a nos promoter (see Fig. 1). Plants transformed with this construct are expected to be resistant to hygromycin, but sensitive to streptomycin, due to the short half-life of the fusion construct, which is based on the short-lived mistargeted OE33. Seeds from these transgenic plants would then be chemically mutagenized with ethylmethane sulfonate (EMS), and mutant seeds selected for the acquisition of streptomycin resistance. At least some of the mutant plants that have become streptomycinresistant are expected to show this phenotype due to stabilization of the fusion protein by a mutation in a gene that encodes a component of the chloroplast proteolytic machinery.

The translation construct is short lived and can confer streptomycin resistance

To test the feasibility of our strategy, two preliminary experiments were carried out. First, we tested whether the fusion protein, composed of the aadA gene product fused to OE33, under control of the SP6 promoter (see Fig. 1), could confer streptomycin resistance to $E$. coli cells. Upon transformation, transformants became resistant to streptomycin whereas non-transformed cells did not (not shown). Second, we tested the import capability and post-import stability of the fusion protein in isolated chloroplasts. The radiolabeled fusion protein was imported into isolated chloroplasts and, similar to its parent construct (Halperin and Adam 1996), was unstable (not shown). Thus, the fusion protein appeared to be suitable for in vivo studies. It was expected to be targeted to chloroplasts, but was not expected to accumulate there due to its short half-life.

Transgenic plants are either sensitive or resistant to streptomycin

Arabidopsis plants were transformed with the transformation construct and seeds were collected. Transformants were selected on agar plates containing hygromycin, and their seeds were collected and further analyzed. As expected, whereas wild-type seeds were sensitive to hygromycin, transgenic seeds were resistant (Fig. 2a). The frequency of transformed seeds was 1:2,000. We anticipated that all transgenic seedlings would be sensitive to streptomycin due to the short a

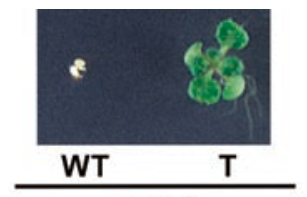

Hyg
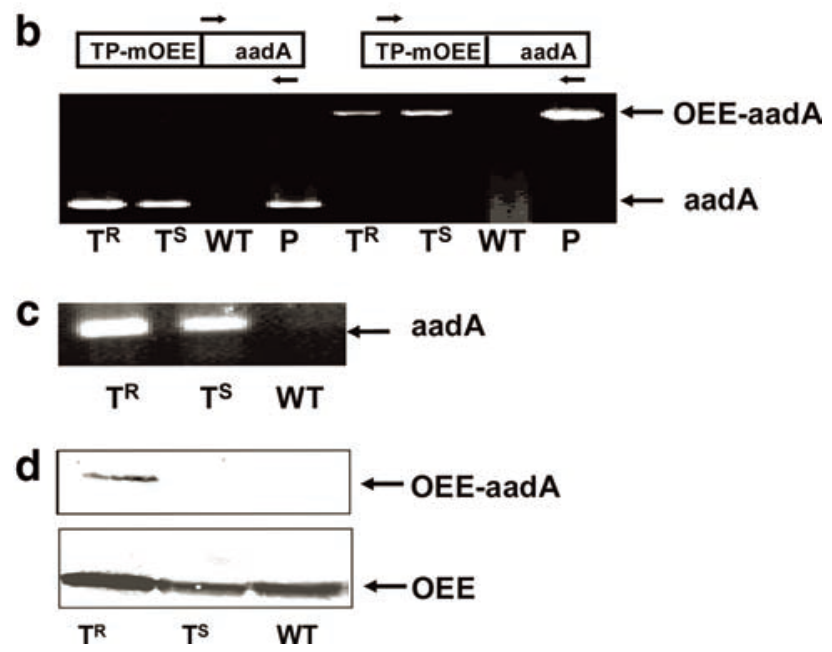

Fig. 2a-d Analysis of transgenic Arabidopsis plants. Wild-type and transgenic plants, selected on plates containing $20 \mathrm{mg} \mathrm{m}^{-1}$ hygromycin, were analyzed. a Resistance of wild-type $(W T)$ and transgenic $(T)$ plants to $20 \mu \mathrm{g} \mathrm{ml}^{-1}$ hygromycin $(H y g)$ and $50 \mu \mathrm{g}$ $\mathrm{ml}^{-1}$ streptomycin $(S t r) . T^{S}$ and $T^{R}$ are transgenic plants sensitive and resistant to streptomycin, respectively. b PCR analysis. DNA was extracted from wild-type $(W T)$ and mutant plants $\left(T^{S}\right.$ or $\left.T^{R}\right)$, and was subjected to PCR with two sets of primers, corresponding to either the Str-resistance gene $(a a d A)$ or a combination of this gene and the fused OE33 (OE33-aadA). Control reactions were carried out on plasmid DNA $(P)$ containing a cDNA encoding the fusion protein. c RT-PCR. RNA was extracted from the above plants and subjected to RT-PCR analysis with a set of primers corresponding to the aadA gene. d Immunoblot analysis. Proteins were extracted from the above plants and subjected to immunoblot analysis with a polyclonal antibody against OE33

half-life of the fusion protein in chloroplasts. In fact, we obtained two populations of transgenic plants: one sensitive to streptomycin and the other resistant (Fig. 2a). Streptomycin-resistant $\left(\mathrm{T}^{\mathrm{R}}\right)$ and streptomycinsensitive $\left(T^{S}\right)$ seedlings were analyzed by PCR. Using two sets of primers, one for the aadA gene only, and the other encompassing the OE33-aadA fusion, we observed that both streptomycin-resistant and sensitive seedlings contained the transgene, whereas wild-type seedlings did not (Fig. 2b). Control reactions with the transformation construct as a template gave products with sizes similar to those observed in transgenic plants, thus supporting our conclusion.

To confirm that the transgene was indeed expressed, RT-PCR was performed with primers for the aadA gene. As shown in Fig. 2c, both streptomycin-sensitive and resistant plants contained the mRNA product of the transgene, whereas wild-type plants did not. Apparently, the streptomycin-resistant plant contained almost twice the transcript found in the streptomycin-sensitive one. Expression at the protein level followed the same 
pattern. Using an antibody against OE33, we could detect this protein in transgenic plants, as well as in wildtype plants as an internal control for quantification. However, whereas wild-type and streptomycin-sensitive plants did not contain the fusion protein, a small amount of it could be detected in the streptomycinresistant transgenic plants (Fig. 2d). Thus, resistance to streptomycin could be correlated with increased levels of expression of the transgene, at both the mRNA and protein levels.

Random mutagenesis of a streptomycin-sensitive transgenic plant

One of the streptomycin-sensitive transgenic plants that demonstrated the highest fertility, designated 6T, was selected for further manipulation. This transgenic line accumulated the transcribed transgene but not the chimeric protein (Fig. 2c, d). Seeds of the plant were subjected to chemical mutagenesis using EMS (see Fig. 1). Following mutagenesis, seeds were germinated and plants were grown to maturity. Mutant seeds $\left(\mathbf{M}_{2}\right)$ were collected from these plants and subjected to selection on agar plates containing both hygromycin and streptomycin. Seedlings demonstrating resistance to both drugs were grown to maturity, and their seeds were collected and subjected to self-fertilization. The homozygous nature of these plants was determined by the resistance of all its germinated seedlings to streptomycin. Figure 3a shows one of the mutants, designated $15 \mathrm{M}$, that acquired resistance to streptomycin, in contrast to its parent plant 6T, which was sensitive. PCR with a pair of specific primers for the OE33 and aadA genes demonstrated that the mutant plant contained the transgene, just like its parent (Fig. 3b). Although 15M plants did not accumulate more transcript of the transgene than $6 \mathrm{~T}$ plants (Fig. 3d), western blot analysis showed that 15M plants had indeed accumulated the fusion protein, unlike the parent plant 6T, which had not (Fig. 3c).

One possible explanation for accumulation of the fusion protein in mutant plants could be an effect of the chemical mutagenesis on the expression of the transgene itself. Thus, to test this possibility, we carried out a northern blot analysis with a probe specific to the OE33 gene. This probe allowed simultaneous detection of both the fusion constructs mRNA and the endogenous OE33 transcript as a loading control. As expected, wild-type plants as well as the parent transgenic plant 6T and two mutant plants expressed high levels of OE33 (Fig. 3d; shorter exposure of this blot showed equal expression of OE33 in all plants). The 6T plant and its mutants expressed the fusion construct as well (Fig. 3d). However, whereas one of the mutants, $2 \mathrm{M}$, became resistant to streptomycin, probably due to a high level of expression of the fusion construct, expression of this construct in the $15 \mathrm{M}$ mutant was even lower than that in $6 \mathrm{~T}$. Thus, it is likely that the accumulation of fusion protein shown in Fig. 3c resulted from decreased degradation of the a

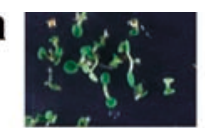

$6 \mathrm{~T}$

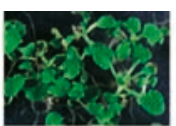

$15 \mathrm{M}$

\section{Hyg + Str}

b
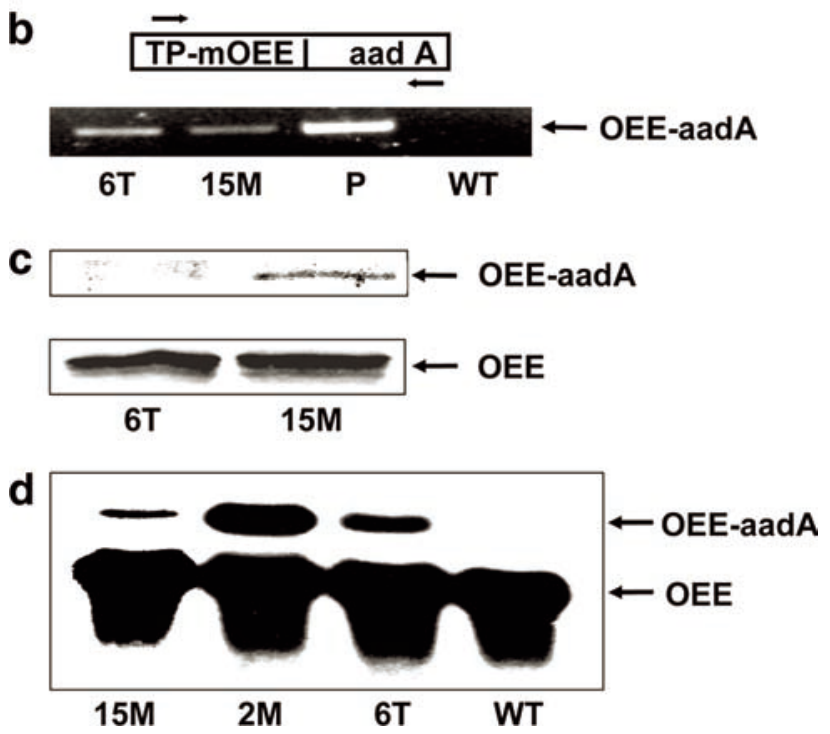

Fig. 3a-d Analysis of mutant Arabidopsis plants. Seeds from one of the transgenic plants described in Fig. 2 (6T), that showed sensitivity to streptomycin, were subjected to EMS mutagenesis. Mutant plants (15M and $2 \mathrm{M})$ were further analyzed. a Resistance of transgenic (6T) and mutant $(15 \mathrm{M})$ plants to hygromycin $(\mathrm{Hyg})$ and streptomycin $(S t r)$. b PCR analysis. DNA was extracted from wild-type $(W T)$, transgenic $(6 T)$ and mutant $(15 M)$ plants, and was subjected to PCR with a set of primers encompassing the OE33aadA transgene. A control reaction with the plasmid DNA $(P)$ containing the transgene was also performed. c Immunoblot analysis. Proteins were extracted from the above plants and subjected to immunoblot analysis with a polyclonal antibody against OE33. d RNA blot analysis. Total RNA was extracted from the above plants and subjected to RNA blot analysis with a DNA probe prepared from the OE $33 \mathrm{cDNA}$. The locations of transcripts corresponding to the endogenous OE33 and to the product of the transgene are indicated. $2 M$ Transgenic mutant plant No. 2

protein in the mutant plant, rather than from increased expression at the mRNA level.

In vitro protein degradation in chloroplasts of the mutant plant

To further characterize protein degradation in chloroplasts, we prepared lysates from chloroplasts isolated from wild-type and mutant plants. Using Rieske Fe-S protein (RISP) as a substrate as previously described (Ostersetzer and Adam 1997), we tested its stability in these lysates. As expected, about $50 \%$ of the substrate protein was degraded following $2 \mathrm{~h}$ of incubation in the wild-type chloroplast lysate (Fig. 4). Even more efficient degradation, almost $90 \%$ of the protein, was observed when the reaction mixture was supplemented with ATP. In contrast, lysates prepared from the mutant protein 

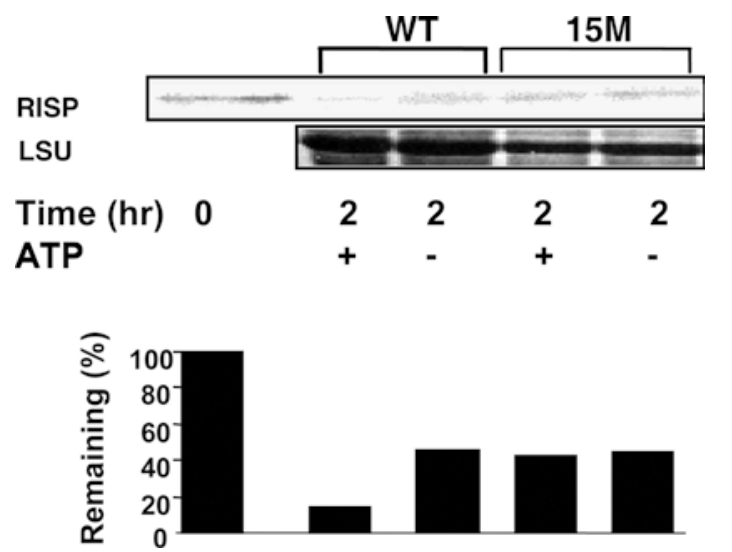

Fig. 4 In-vitro protein degradation in wild-type and mutant Arabidopsis plants. Chloroplasts (5 $\mu$ g chlorophyll) from wild-type $(W T)$ and mutant $(15 M)$ plants were lysed and then incubated with $1000 \mathrm{cpm}$ of radiolabelled, in vitro-translated RISP for $2 \mathrm{~h}$, in the presence or absence of $5 \mathrm{mM}$ ATP. Reaction mixtures were then subjected to SDS-PAGE, and the levels of remaining RISP were visualized (RISP) and quantified (bar graph) using a PhosphorImager (Fuji). Loading controls are presented by the large subunit $(L S U)$ of Rubisco band from the Coomassie blue-stained gel. The presented results are from one out of three experiments with similar results

could support ATP-independent degradation to a similar extent, but ATP-dependent degradation was completely inhibited (Fig. 4). Similar results were observed using $\beta$-casein as a substrate (data not shown). These results supported the notion that the proteolytic machinery in chloroplasts of the mutant plant is indeed impaired.

\section{Genetic analysis of the mutant}

To characterize the genetic nature of the mutation, reciprocal crosses between the homozygous mutant plant $15 \mathrm{M}$ and wild-type plants were carried out. Wild-type plants carry the unknown gene " $\mathrm{A}$ " that is mutated in $15 \mathrm{M}$ plants, and is therefore designated " $\mathrm{A}$ ". In addition, the mutant plants carry the transgene, containing linked genes for both hygromycin and streptomycin resistance ("H-S"; Fig. 5). If the mutation were in a plastid gene, $F_{1}$ seeds from a cross between $15 \mathrm{M}$ and the wild type would yield a 4:0 ratio between streptomycinresistant and sensitive seedlings due to maternal inheritance of plastids. The reciprocal cross would yield no resistant plants because plastids are not carried on by pollen. Since none of the crosses gave these ratios, we concluded that the mutation was in a nuclear gene. To further analyze the mutant, we tested segregation of $\mathrm{F}_{2}$ seeds with respect to hygromycin and streptomycin resistance. The different genotypes expected from this cross are presented in Fig. 5. All seeds carrying either single or double alleles of the transgene $(\mathrm{H}-\mathrm{S})$ are expected to be resistant to hygromycin. However, although the hygromycin- and streptomycin-resistance genes are linked in the transgene, streptomycin resis-

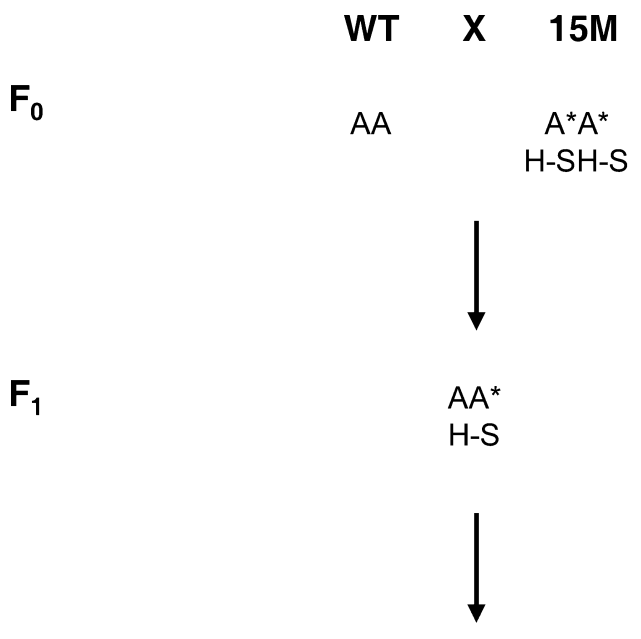

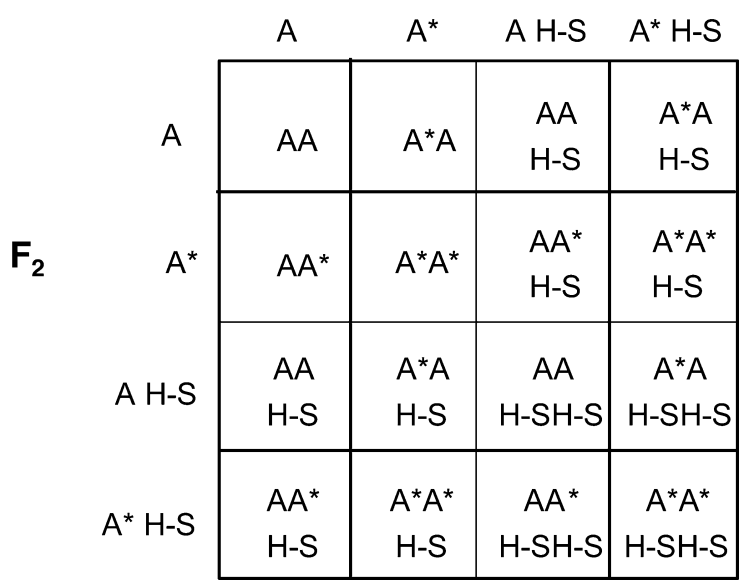

Fig. 5 Genotypes of wild-type and mutant Arabidopsis plants and their progeny. Wild type $(W T)$ and mutant $(15 M)$ plants were crossed, and $F_{1}$ plants were self-crossed again to yield the $F_{2}$ generation. $A$ and $A^{*}$ represent the wild-type and mutant alleles of an unknown gene, respectively, where a mutation is expected to stabilize the product of the streptomycin-resistance gene. $H-S$ represents the linked hygromycin- and streptomycin-resistance genes of the transgene

tance should be manifested only in plants carrying, in addition to the transgene, a mutant allele of the unknown gene A that stabilizes the product of the streptomycin-resistance gene. The calculated ratios between the different phenotypes in the $\mathrm{F}_{2}$ generation are presented in Table 1 . If the mutation in gene $\mathrm{A}$ is recessive, only 3 out of 16 seeds are expected to be resistant to both hygromycin and streptomycin $\left(\mathrm{A}^{*} / \mathrm{A}^{*}\right.$ seedlings), whereas 9 out of 16 seeds will be resistant to hygromycin, but sensitive to streptomycin. The opposite is expected if the mutation is dominant. As shown in Table 1, when resistance to either hygromycin or streptomycin was scored, only 70 out of 384 seedlings demonstrated resistance to both antibiotics. Resistance to hygromycin was close to the expected ratio of 0.75 . Thus, it was concluded that the mutation conferring resistance to streptomycin is a recessive mutation in a single nuclear gene that is not linked to the transgene itself. 
Phenotypic effects of the mutation

To characterize the phenotypic effects of the mutation, wild-type and mutant seeds were germinated and grown at $22{ }^{\circ} \mathrm{C}$, under a light intensity of $100 \mu \mathrm{mol}$ photons $\mathrm{m}^{-2} \mathrm{~s}^{-1}$. Since no obvious differences were observed between the two groups, we subjected plants to either low $\left(5^{\circ} \mathrm{C}\right)$ or high temperature $\left(42^{\circ} \mathrm{C}\right)$, and high light intensity $\left(500 \mu \mathrm{mol}\right.$ photons $\left.\mathrm{m}^{-2} \mathrm{~s}^{-1}\right)$. Once again, no major differences were observed between mutant and wild-type plants in response to these conditions. The only visible difference was a shorter time required for flowering in plants grown at $16{ }^{\circ} \mathrm{C}: 47$ vs. 51 days in mutant and wild-type plants, respectively, whereas at higher growth temperature, there was no such difference. Further examination of chlorophyll concentration, protein profiles on single-dimension SDS gels and in vivo pulse-chase labeling experiments in mature leaves revealed no differences either. Confocal microscopy of emerging cotyledons from wild-type and mutant plants revealed differences in the distribution of the autofluorescence. Whereas chlorophyll fluorescence from wildtype cotyledons was evenly distributed throughout the chloroplasts, fluorescence from the mutant was more intense in defined regions within the chloroplast (Fig. 6), showing a striated pattern.

Table 1 Phenotypes of $\mathrm{F}_{2}$ progeny from a cross between wild-type and mutant Arabidopsis thaliana plants. Calculated and observed ratios between the different phenotypes of $\mathrm{F}_{2}$ progeny are presented. The calculated ratios, for either a recessive or a dominant mutation, are based on the different genotypes presented in Fig. 5. All $\chi^{2}$ values were in the range of $0.995>P>0.5$. Str, streptomycin; $H y g$, hygromycin; ${ }^{R}$, resistant; ${ }^{S}$, sensitive

\begin{tabular}{llll}
\hline Phenotype & \multicolumn{2}{l}{ Ratio (resistant/sensitive:total seedlings) } \\
\cline { 2 - 3 } & \multicolumn{2}{l}{ Calculated } & Observed \\
\cline { 2 - 3 } & Dominant & Recessive & \\
\hline $\mathrm{Str}^{\mathrm{R}}$ & $9: 16=0.562$ & $3: 16=0.187$ & $70: 384=0.182$ \\
$\mathrm{Str}^{\mathrm{S}}$ & $7: 16=0.437$ & $13: 16=0.812$ & $314: 384=0.818$ \\
$\mathrm{Hyg}^{\mathrm{R}}$ & $12: 16=0.750$ & $12: 16=0.750$ & $23: 29=0.793$ \\
$\mathrm{Hyg}^{\mathrm{S}}$ & $4: 16=0.250$ & $4: 16=0.250$ & $6: 29=0.207$ \\
\hline
\end{tabular}
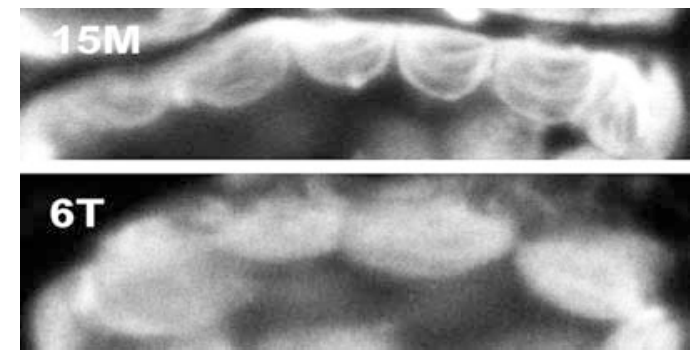

Fig. 6 Confocal microscopy of wild-type and mutant Arabidopsis cotyledons. Cotyledons from transgenic (6T) and mutant (15M) plants were first fixed for $2 \mathrm{~h}$ in $8 \%(\mathrm{v} / \mathrm{v})$ formaldehyde and $5 \%(\mathrm{v} /$ v) DMSO, in a PEM buffer (see Materials and Methods), and then subjected to confocal microscopy
Effect of growth at low temperature on photosynthesis

To monitor possible functional effects of the mutation, we carried out an analysis of photosynthetic parameters by measuring chlorophyll fluorescence. Looking at the maximum quantum yield of photosystem II electron transport $\left(F_{\mathrm{V}} / F_{\mathrm{M}}\right)$, no major differences could be observed between mutant and wild-type plants after exposure to either high light intensity or high temperature (Fig. 7a). Only a slight reduction of 7\% was observed in the mutant cold-stressed plants (Fig. 7b). Further fluorescence analysis of these latter plants revealed a larger reduction of $18 \%$ in the quantum yield efficiency of photosystem II (the "Y" parameter) of mutant plants compared with the wild type (Fig. 7b).
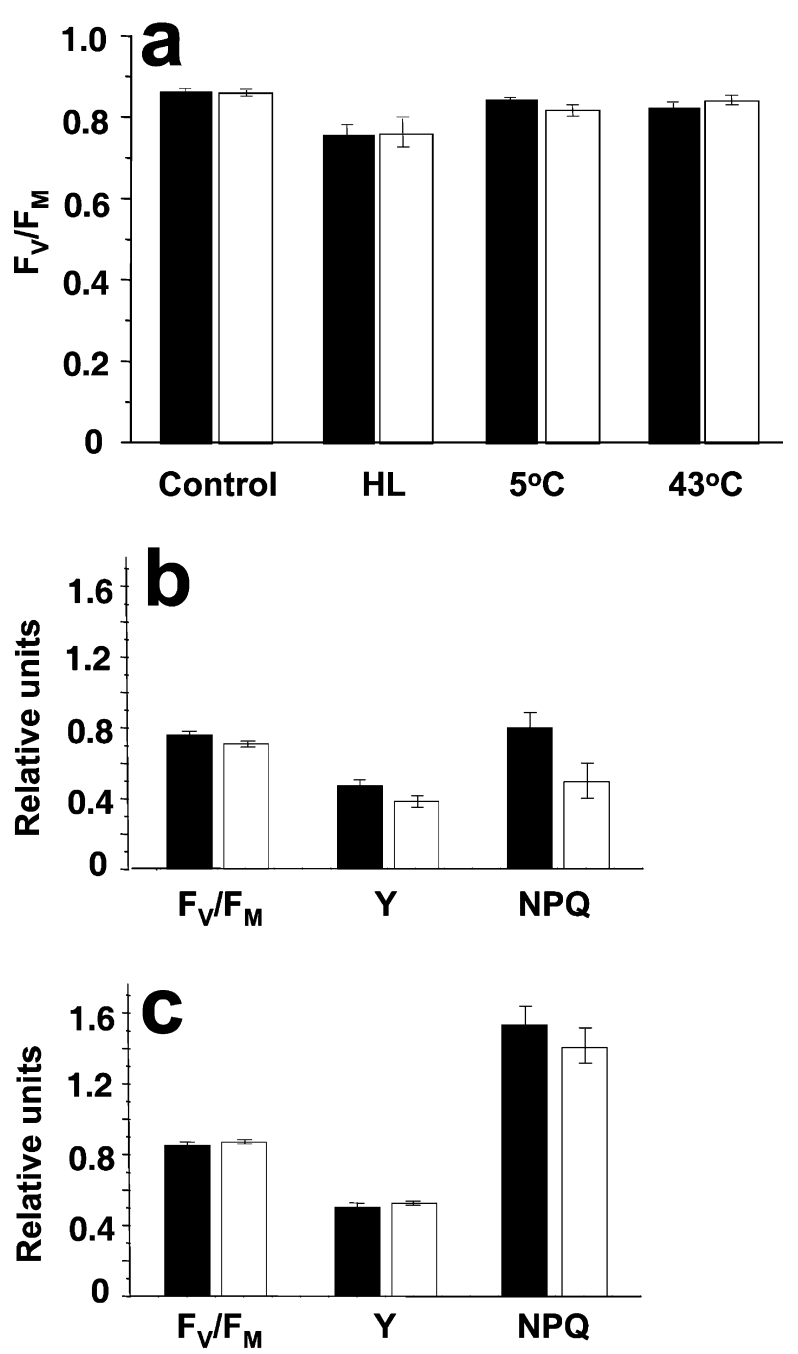

Fig. 7a-c Chlorophyll fluorescence from wild-type and mutant Arabidopsis plants. Six-week-old wild-type (filled bars) and mutant (open bars) plants were exposed to either high light intensity $(H L)$ or $5{ }^{\circ} \mathrm{C}$ for 7 days, or $43^{\circ} \mathrm{C}$ for $3 \mathrm{~h}$. After these treatments, the maximum quantum yield of photosystem II electron transport $\left(F_{\mathrm{V}} /\right.$ $F_{\mathrm{M}}$ ) was monitored by chlorophyll measurements (a). Photosystem II yield $(Y)$ and non-photochemical quenching (NPQ) were also monitored in cold-stressed (b) and control (c) plants. Values represent means \pm SE of eight independent measurements 
Non-photochemical quenching (NPQ) was calculated to be $38 \%$ lower in the mutant plants than in the wild-type plants (Fig. 7b). Similar measurements conducted on plants that were kept continuously at $22{ }^{\circ} \mathrm{C}$ revealed no differences between wild-type and mutant plants (Fig. 7c), suggesting that, following long exposure to low temperature, mutant plants suffer from inhibition at the heat-dissipation mechanism and/or at photosynthetic electron transport. A similar effect was observed when the transgenic line 6T was compared to the mutant line $15 \mathrm{M}$ (data not shown).

\section{Discussion}

The importance of chloroplast protein degradation for maintenance of the photosynthetic machinery, especially under changing environmental conditions, has been long recognized. However, biochemical attempts to purify and identify the relevant proteases have been largely unsuccessful. Application of indirect molecular approaches in recent years have led to the identification and characterization of chloroplast proteases, all of which are homologues of known bacterial proteases (for review, see Adam et al. 1998; Adam 2001). To expand our ability to identify unknown components of the chloroplast proteolytic machinery, we took a genetic approach in generating transgenic mutant plants impaired in their ability to degrade chloroplast proteins. This positive-selection approach was based on a previous successful study, in which mutants in cytoplasmic ubiquitin-mediated protein degradation were generated and characterized, and the affected gene was cloned (Bachmair et al. 1993; Potuschak et al. 1998).

Initial characterization of our transgenic plants bearing the reporter construct revealed that resistance to our selection marker, streptomycin, was correlated with the level of expression of the transgene (Fig. 2). Random mutagenesis of seeds from one of the streptomycinsensitive transformants, followed by selection on streptomycin, identified a number of mutants in which the product of the transgene was apparently stabilized (Fig. 3). Whereas in at least one such mutant (2M) resistance could be correlated with higher levels of the transgene mRNA, in another mutant (15M), the transgene mRNA level was comparable to that of the parent transgenic plant, suggesting that degradation of the chimeric protein in the mutant was indeed lower. This conclusion was further supported by an in vitro proteindegradation assay (Fig. 4). Reciprocal crosses between the mutant and wild-type plants, followed by scoring the progeny for antibiotic resistance, suggested that the mutation was recessive, in a single nuclear gene (Fig. 5, Table 1).

When grown under different environmental conditions, the mutant could not be distinguished from the wild-type plant. However, confocal microscopy revealed different patterns of autofluorescence (Fig. 6). Whereas chlorophyll fluorescence of wild-type chloroplasts appeared evenly distributed in the organelle, fluorescence in the mutant was restricted to well-defined regions. Although the reason for this pattern is not clear to us, it does suggest that functional aspects of chloroplasts might be impaired as well. Measuring chlorophyll fluorescence from leaves revealed that upon exposure to cold stress, the mutant displays lower values of photosynthetic parameters (Fig. 7). Only a slight reduction of less than $10 \%$ in the maximum quantum yield of photosystem II electron transport was observed in mutant plants, but a larger decrease in the quantum yield efficiency of photosystem II, almost $20 \%$, was observed in the mutant compared to the wild type. An even larger difference, a reduction of almost $40 \%$, was observed in the NPQ of mutant plants. In contrast, no differences were observed between wild-type and mutant plants grown at $22{ }^{\circ} \mathrm{C}$. These results suggest dysfunction of photoprotection components and inhibition of photosynthetic electron transport. It is well documented that low temperatures enhance photoinhibition (Krause 1994; Long et al. 1994). Photoinhibition has been traditionally associated with damage to photosystem II, but evidence for inactivation of photosystem I under photoinhibitory conditions has also been reported. Interestingly, inactivation of photosystem I has been observed at low temperatures in both cold-sensitive and cold-tolerant species (Terashima et al. 1994; Sonoike 1996b; Tjus et al. 1999), consistent with the observation of reduced NPQ in mutant plants only after exposure to low temperature.

Why the mutant plant is more sensitive than the wild type to photoinhibition is not yet clear. However, the higher sensitivity to photoinhibition upon cold stress in a mutant plant that is impaired in chloroplast protein degradation makes it tempting to speculate that a mutation in the proteolytic machinery impedes a repair mechanism necessary to overcome damage incurred to the photosynthetic machinery during photoinhibition. As already mentioned, we suspect that the reduced NPQ observed in the mutant after cold stress results from inhibition of a heat-dissipation mechanism that might later on inhibit electron transport and photosynthesis. Damage and degradation of photosystem reaction center subunits upon photoinhibition have been reported in the past (Sonoike 1996a; Tjus et al. 1999; Melis 1999). A repair mechanism for inactivated photosystem centers involves proteolytic degradation of damaged subunits followed by insertion of newly synthesized components. A mutation in the chloroplast proteolytic machinery may result in failure to remove damaged subunits, and thus inhibition of repair. Since proteolytic degradation is an enzymatic process, its inhibition may be more pronounced at low temperatures than at higher ones.

The mild nature of the mutation and the lack of a visual phenotype raise the question of why a mutation in a supposedly important cellular system is not manifested in a more severe phenotype. One possible explanation is that the products of other genes compensate for the affected one. This view is supported by the fact that 
known chloroplast proteases occur in plant genomes in multiple isomers: $\mathrm{ClpC}$ is encoded by two closely related genes, $\mathrm{ClpP}$ is found in six copies, FtsH appears to have 12 isomers and DegP has 14 (Adam et al. 2001; Adam and Clarke 2002; Sokolenko et al. 2002). How many of these are indeed found within chloroplasts is not yet clear. But it is certain that for each protease more than one isomer does exist. Although the extent of redundancy in function within these families is also unknown, the possibility for compensation does seem likely, in view of the recent finding that a variegated phenotype of Arabidopsis was linked to mutations in the fts $\mathrm{H} 2$ gene (Chen et al. 2000; Takechi et al. 2000). Thus, although it is not clear how a single genotype is translated into two adjacent phenotypes, white and green sectors, this finding suggests that a mutation in one protease gene can be compensated for by other related genes.

Another possible explanation is related to the nature of the mutated system. Since one of the functions of the proteolytic machinery is to dispose of damaged, nonfunctional proteins, it may take time for the accumulation of damaged proteins to reach a level that is manifested phenotypically. An interesting, though remote analogy might be the case of heredity spastic paraplegia. This is a late-onset human illness that is caused by mutations in a mitochondrial ATP-dependent protease (Casari et al. 1998), a homologue of the FtsH protease found in bacteria, yeast mitochondria and plant chloroplasts. It affects only the longest axons of the central nervous system, and takes years to show clinical symptoms. Thus, it is likely that only under certain combinations of time and cell types are compensating proteins insufficient to overcome the effects of the mutation, allowing symptoms to eventually appear. A similar sequence of events may be occurring in our mutant, in that only after exposure to cold stress that might reduce the efficiency of the compensating mechanisms does the mutant show physiological defects. Further clarification of this point awaits identification of the affected gene.

Acknowledgements We thank Dr. Klaudia Brix for skillful help with confocal microscopy, Dr. Victor Raskin for excellent help and advice on chlorophyll fluorescence measurements, and Dr. Nir Ohad and Profs. Avi Levy and Itzhak Ohad for insightful discussions. This work was supported by grants from The Austrian Friends of The Hebrew University (to A.B. and Z.A.), the Israel Ministry of Science (to Z.A.), and the US-Israel Binational Agricultural Research and Development Fund-BARD (to Z.A.).

\section{References}

Adam Z (1996) Protein stability and degradation in chloroplasts. Plant Mol Biol 32:773-783

Adam Z (2000) Chloroplast proteases: possible regulators of gene expression? Biochimie 82:647-654

Adam Z (2001) Chloroplast proteases and their role in photosynthesis regulation. In: Aro E-M, Andersson B (eds) Regulation of photosynthesis. Kluwer, Dordrecht, pp 265-276

Adam Z, Clarke AK (2002) Cutting edge of chloroplast proteolysis. Trends Plant Sci 7:451-456
Adam Z, Halperin T, Itzhaki H, Lindahl M, Ostersetzer O (1998). The proteolytic machinery of chloroplasts: homologues of bacterial proteases. In: Garab G (ed) Photosynthesis: mechanisms and effects, vol 3. Kluwer, Dordrecht, pp 1871-1876

Adam Z, Adamska I, Nakabayashi K, Ostersetzer O, Haussuhl K, Manuell A, Vallon O, Rodermel SR, Shinozaki K, Clarke AK (2001) Chloroplast and mitochondrial proteases in Arabidopsis. A proposed nomenclature. Plant Physiol 125:1912-1918

Andersson B, Aro E-M (1997) Proteolytic activities and proteases of plant chloroplasts. Physiol Plant 100:780-793

Bachmair A, Becker F, Schell J (1993) Use of a reporter transgene to generate Arabidopsis mutants in ubiquitin-dependent protein degradation. Proc Natl Acad Sci USA 90:418-421

Bechtold N, Ellis J, Pelletier G (1993) In planta Agrobacterium mediated gene transfer by infiltration of adult Arabidopsis thaliana plants. C R Acad Sci Paris Life Sci 316:1194-1199

Becker D (1990) Binary vectors which allow the exchange of plant selectable markers and reporter genes. Nucleic Acids Res 18:203

Casari G, De Fusco M, Ciarmatori S, Zeviani M, Mora M, Fernandez P, De Michele G, Filla A, Cocozza S, Marconi R, Durr A, Fontaine B, Ballabio A (1998) Spastic paraplegia and OXPHOS impairment caused by mutations in paraplegin, a nuclear-encoded mitochondrial metalloprotease. Cell 93: 973-983

Chassin Y, Kapri-Pardes E, Sinvany G, Arad T, Adam Z (2002) Expression and characterization of the thylakoid lumen protease DegP1 from Arabidopsis thaliana. Plant Physiol 130:857864

Chen M, Choi Y, Voytas DF, Rodermel S (2000) Mutations in the Arabidopsis VAR2 locus cause leaf variegation due to the loss of a chloroplast FtsH protease. Plant J 22:303-313

Clarke AK (1999) ATP-dependent Clp proteases in photosynthetic organisms - a cut above the rest! Ann Bot 83:593-599

Gottesman S (1996) Proteases and their targets in Escherichia coli. Annu Rev Genet 30:465-506

Gottesman S, Wickner S, Maurizi MR (1997) Protein quality control: triage by chaperones and proteases. Genes Dev 11:815823

Halperin T, Adam Z (1996) Degradation of mistargeted OEE33 in the chloroplast stroma. Plant Mol Biol 30:925-933

Halperin T, Ostersetzer O, Adam Z (2001) ATP-dependent association between subunits of Clp protease in pea chloroplasts. Planta 213:614-619

Haussuhl K, Andersson B, Adamska I (2001) A chloroplast DegP2 protease performs the primary cleavage of the photodamaged D1 protein in plant photosystem II. EMBO J 20:713-722

Hershko A, Ciechanover A (1998) The ubiquitin system. Annu Rev Biochem 67:425-479

Itzhaki H, Naveh L, Lindahl M, Cook M, Adam Z (1998) Identification and characterization of DegP, a serine protease associated with the luminal side of the thylakoid membrane. J Biol Chem 273:7094-7098

Koncz C, Martini N, Mayerhofer R, Koncz-Kalman Z, Korber H, Redei GP, Schell J (1989) High-frequency T-DNA-mediated gene tagging in plants. Proc Natl Acad Sci USA 86:8467-8471

Krause G (1994) Photoinhibition induced by low temperatures. In: Baker NR, Bowyer JR (eds) Photoinhibition of photosynthesis from molecular mechanisms to the field. Bios Scientific, Oxford, pp 331-348

Krysan PJ, Young JC, Tax F, Sussman MR (1996) Identification of transferred DNA insertions within Arabidopsis genes involved in signal transduction and ion transport. Proc Natl Acad Sci USA 93:8145-50

Langer T (2000) AAA proteases: cellular machines for degrading membrane proteins. Trends Biochem Sci 25:247-251

Langer T, Neupert W (1996) Regulated protein degradation in mitochondria. Experientia 52:1069-1076

Lindahl M, Tabak S, Cseke L, Pichersky E, Andersson B, Adam Z (1996) Identification, characterization, and molecular cloning of a homologue of the bacterial FtsH protease in chloroplasts of higher plants. J Biol Chem 271:29329-29334 
Lindahl M, Spetea C, Hundal T, Oppenheim AB, Adam Z, Andersson B (2000) The thylakoid FtsH protease plays a role in the light-induced turnover of the photosystem II D1 protein. Plant Cell 12:419-431

Long SP, Humphries S, Falkowski PG (1994) Photoinhibition of photosynthesis in nature. Annu Rev Plant Physiol Plant Mol Biol 45:633-662

Majeran W, Wollman F-A, Vallon O (2000) Evidence for a role of $\mathrm{ClpP}$ in the degradation of the chloroplast cytochrome $\mathrm{b}_{6} \mathrm{f}$ complex. Plant Cell 12:137-149

Melis A (1999) Photosystem-II damage and repair cycle in chloroplasts: what modulates the rate of photodamage? Trends Plant Sci 4:130-135

Ostersetzer O, Adam Z (1997) Light-stimulated degradation of an unassembled Rieske FeS protein by a thylakoid-bound protease: the possible role of the FtsH protease. Plant Cell 9:957-965

Peltier J-B, Ytterberg J, Liberles DA, Roepstorff P, van Wijk KJ (2001) Identification of a $350 \mathrm{kDa}$ ClpP protease complex with 10 different Clp isoforms in chloroplasts of Arabidopsis thaliana. J Biol Chem 276:16318-16327

Potuschak T, Stary S, Schlogelhofer P, Becker F, Nejinskaia V, Bachmair A (1998) PRT1 of Arabidopsis thaliana encodes a component of the plant N-end rule pathway. Proc Natl Acad Sci USA 95:7904-7908

Prasil O, Adir N, Ohad I (1992) Dynamics of photosystem II: mechanism of photoinhibition and recovery processes. In: Barber $\mathbf{J}$ (ed) Topics in photosynthesis. Elsevier, Amsterdam, pp 295-348

Shanklin J, Dewitt ND, Flanagan JM (1995) The stroma of higher plant plastids contain $\mathrm{ClpP}$ and $\mathrm{ClpC}$, functional homologs of Escherichia coli ClpP and ClpA: an archetypal two-component ATP-dependent protease. Plant Cell 7:1713-1722
Sokolenko A, Pojidaeva E, Zinchenko V, Panichkin V, Glaser VM, Herrmann RG, Shestakov SV (2002) The gene complement for proteolysis in the cyanobacterium Synechocystis sp. PCC 6803 and Arabidopsis thaliana chloroplasts. Curr Genet 41:291-310

Sonoike K (1996a) Photoinhibition of photosystem I: its physiological significance in the chilling sensitivity of plants. Plant Cell Physiol 37:239-247

Sonoike K (1996b) Degradation of PsaB gene product, the reaction center subunit of photosystem I, is caused during photoinhibition of photosystem I: possible involvement of active oxygen species. Plant Sci 115:157-164

Tait RC, Rempel H, Rodriguez RL, Kadu CI (1985) The aminoglycoside resistance operon of the plasmid pSa: nucleotide sequence of the streptomycin-spectinomycin resistance gene. Gene 36:97-104

Takechi K, Sodmergen, Murata M, Motoyoshi F, Sakamoto W (2000) The YELLOW VARIEGATED (VAR2) locus encodes a homologue of FtsH, an ATP-dependent protease in Arabidopsis. Plant Cell Physiol 41:1334-1346

Terashima I, Funayama S, Sonoike K (1994) The site of photoinhibition in leaves of Cucumis sativa L. at low temperatures is photosystem I, not photosystem II. Planta 193:300-306

Tjus SE, Moller BL, Scheller HV (1999) Photoinhibition of photosystem I damages both reaction center proteins PSI-A and PSI-B and acceptor-side located small photosystem I polypeptides. Photosynth Res 60:75-86

Topfer R, Matzeit V, Gronenborn B, Schell J, Steinbiss HH (1987) A set of plant expression vectors for transcriptional and translational fusions. Nucleic Acids Res 15:5890.

Wickner S, Maurizi MR, Gottesman S (1999) Posttranslational quality control: folding, refolding, and degrading proteins. Science 286:1888-1893 